\title{
A Power Independent Detection Method for Ultra Wide Band (UWB) Impulse Radio Networks
}

\author{
Alaeddine El Fawal and Jean-Yves Le Boudec \\ School of Computer and Communication Sciences \\ Ecole Polytechnique Fédérale de Lausanne (EPFL) \\ \{alaeddine.elfawal, jean-yves.leboudec\}@epfl.ch
}

\begin{abstract}
We propose a novel detection method for noncoherent synchronization (signal acquisition) in multi-access UWB impulse radio (IR) networks. It is designed to solve the IUI (InterUser Interference) that occurs in some ad-hoc networks where concurrent transmissions are allowed with heterogeneous power levels. In such scenarios, the conventional detection method, which is based on correlating the received IR signal with a Template Pulse Train (TPT), does not always perform well. Our proposal has similar complexity as the conventional method. We evaluate its performance with the Line Of Sight (LOS) office indoor channel model proposed by the IEEE P802.15.4a study group and find that the improvement is significant.
\end{abstract}

\section{INTRODUCTION}

We propose a novel detection method, called PID (Power Independent Detection) method, for non-coherent synchronization in multi-access Ultra Wide Band (UWB) Impulse Radio (IR) networks. To understand what we mean by detection method, let us define the following terminology. We consider the synchronization of one receiver to one sender (also called signal acquisition). We are interested in methods based on the correlation of the IR signal with a Template Pulse Train (TPT). Such methods involve two ingredients: (1) the detection, which correlates the received signal with a TPT and (2) the search algorithm, which shifts the TPT. We focus on detection. Our proposal aims at solving the extreme Inter-User Interference (IUI) case (near-far problem), when there are multiple interfering transmitters, asynchronous transmissions and heterogeneous power levels. This occurs for example in the presence of multiple interfering piconets, or in purely ad-hoc networks that allow concurrent transmissions, always at full power [1], [2]. In such scenarios the conventional detection method faces a certain failure. Our PID method solves the problem without any additional complexity overhead, e.g. for a digital receiver, it employs the same sampling frequency and number of operations as the conventional detection method. Unlike the conventional detection method, the PID method splits the correlation into elementary correlations, each one corresponds to one pulse in the TPT. Then, two threshold checks are performed. The first is to detect pulses whereas the second is to detect the signal based on the number of detected pulses. To evaluate the performance of the PID method, we propose a hybrid method combining analysis and simulation that is carried out according to the

The work presented in this paper was supported (in part) by the National Competence Center in Research on Mobile Information and Communication Systems (NCCR-MICS), a center supported by the Swiss National Science Foundation under grant number 5005-67322.
Line Of Sight (LOS) office indoor channel model proposed by the IEEE P802.15.4a study group [3]. The results presented in the end show a significant improvement compared to the conventional detection method.

The conventional detection method (detailed in III-A) has been recently adopted in a large number of references, in combination with a variety of search algorithms; Some search algorithms are adequate for fine grained synchronization (e.g. serial [4]) or for coarse synchronization (e.g. "Look and Jump", Bit reversal [5]-[7], sequential block search [8] and n-scaled acquisition algorithms [9]). The conventional detection method suffers from the near-far problem with all these search algorithms. The PID method, is designed to replace it and solve the near-far problem with all the search algorithms. Further, the PID method can be generalized to all correlation-based synchronization methods (e.g. conventional one and others such as Differential Detector [10]-[12]) and it can be even used with a rake detector as proposed in [13], which do not operate well in case of near-far problem.

\section{Model AND Assumption:}

An IR signal consists of trains of very short pulses to the order of a nanosecond or even a sub-nanosecond. In this paper we consider a Time Hopping (TH) physical layer proposed by Win-Scholtz [14]. TH is ensured using a pseudo-random code of length $L_{c}$. Such a physical layer can employ several modulation schemes such as BPSK (Binary Phase Shift Keying), PPM (Pulse Position Modulation), PAM (Pulse Amplitude Modulation); we do not have to specify a modulation scheme here since there is no data transmitted during the synchronization period and thus the signal we treat is not modulated. The transmitted signal of the $m^{\text {th }}$ user is:

$$
\begin{aligned}
s^{(m)} & =A^{(m)} \sum_{j} \sum_{k=1}^{L_{c}} \\
& p\left(t-\left(c_{k}^{(m)}-1\right) T_{c}-(k-1) T_{f}-j T_{s}-\tau_{X}^{(m)}\right)
\end{aligned}
$$

where $p(t)$ is the second derivative of the Gaussian pulse (Appendix $\left.{ }^{1}\right), A^{(m)}$ is to indicate the signal amplitude, $T_{c}$ is the chip duration, $c_{k}^{(m)}$ is the $k^{t h}$ element of the $m^{t h}$ user code, i.e. the number of the chip that corresponds to the pulse position in the $k^{t h}$ frame of a $m^{t h}$ user sequence, $T_{f}=N_{c} \times T_{c}$ is the frame duration where $N_{c}$ is the number of chips in one frame,

\footnotetext{
${ }^{1}$ All appendices cited in this paper belong to our technical report [15].
} 
$T_{s}$ is the sequence duration, that is $T_{s}=T_{f} \times L_{c}$ and $\tau_{X}^{(m)}$ is the transmission start time. We assume that the pulse width and the chip duration are equal.

For our results, we consider the Saleh-Valenzuela (SV) channel model adopted in [3]. For simplicity, we express its impulse response using the well-known tapped delay line expression:

$$
h(t)=\sum_{l=1}^{L} a_{l} \delta\left(t-t_{l}\right)
$$

where $\delta(t)$ denotes the Dirac impulse, $t_{l}$ the signal delay along the $l^{t h}$ path and $a_{l}$ is a real propagation coefficient that includes the channel attenuation and the polarity of the signal along the $l^{\text {th }}$ path. Then the received signal is given by:

$$
r(t)=\sum_{m=1}^{M} \sum_{l=1}^{L} a_{l}^{(m)} s^{(m)}\left(t-t_{l}^{(m)}\right)+n(t)
$$

where $\mathbf{M}$ is the number of users in the network and $n(t)$ is the White Gaussian noise.

Assume that the receiver is interested in detecting the signal sent by the first user. Then, the objective of the synchronization methods that use either the PID or the conventional detection methods is to detect whether the first user is transmitting or not, and if he is transmitting, they find the arrival time of one sequence in the first user signal according to one of the multipath components, i.e. they find one value of $\left\{\left(\tau_{X}^{(1)}+j T_{s}+t_{l}^{(1)}\right), l=1, \ldots, L, j=0,1, \ldots\right\}$, let $\tau_{0}$ be the found value. Further it detects the sign of the corresponding $a_{l}$.

\section{Conventional Detection Method}

\section{A. Description}

As it is explained in section I, we consider synchronization methods that involve two ingredients: the detection and the search algorithm. With the conventional detection method, the received IR signal is correlated with a TPT, which is a replica of the sequence used by the first user and which is given by:

$$
s_{T P T}(t)=\sum_{k=1}^{L_{c}} p\left(t-\left(c_{k}^{(1)}-1\right) T_{c}-(k-1) T_{f}\right)
$$

The idea behind the correlation is to compare the TPT with the received impulse radio signal, which may or may not have the identical pattern of pulses as the TPT. Then a threshold check is performed on the output of the correlation ( $\beta$ in 5 ) to detect whether there is a match (an alignment) between the TPT and the received IR signal.

The role of the search algorithm is to shift the TPT with predefined time offsets so that the TPT is placed at various locations in time as compared to the received impulse radio signal until a match is obtained between them, i.e. they are aligned. The output of the cross-correlator is:

$$
\beta=\int_{\sum_{i=1}^{n} \text { Offset }_{i}}^{\sum_{i=1}^{n} \text { Offset }_{i}+T_{s}} r(t) \times s_{T P T}\left(t-\sum_{i=1}^{n} \text { Offset }_{i}\right) \mathrm{d} t
$$

where $n$ is the current shift number and Offset ${ }_{i}$ is the time offset at the $i^{t h}$ shift of the TPT. 5 is known in the literature as a coherent integration, but in this paper we refer to it as a correlation between the TPT and the received IR signal (note that we do not assume that the receiver knows the channel). The receiver gets synchronized with the transmitter at the $n^{\text {th }}$ offset if $\sum_{i=1}^{n}$ Offset $_{i}$ is equal to one value of the set $\left\{\left(\tau_{X}^{(1)}+j T_{s}+t_{l}^{(1)}\right), l=1, \ldots, L, j=0,1, \ldots\right\}$, and thus $\tau_{0}=\sum_{i=1}^{n}$ Offset $_{i}$. Notice that, according to 4,5 can be inter-

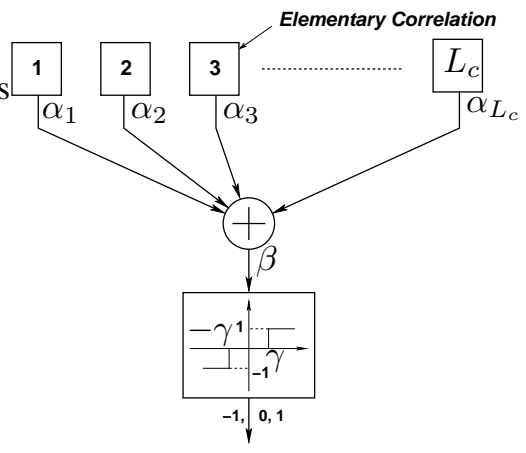

Fig. 1. The conventional detection method can be interpreted as $L_{c}$ elementary cross-correlations. Block i, $i=1, \ldots, L_{c}$, presents the correlation of the $i^{t h}$ pulse in the TPT with its corresponding interval.

preted as $L_{c}$ elementary correlations $\left\{\left(\alpha_{k}\right)\right\}, k=1, \ldots, L_{c} . \alpha_{k}$ is the output of the elementary correlation $k$ that corresponds to the $k^{t h}$ pulse in the TPT. We can write:

$$
\beta=\sum_{k=1}^{L_{c}} \alpha_{k}
$$

where:

$$
\begin{aligned}
\alpha_{k}= & \int_{\left(c_{k}^{(1)}-1\right) T_{c}+(k-1) T_{f}+\sum_{i=1}^{n} \operatorname{Offset}_{i}}^{\left(c_{k}^{(1)}-1\right) T_{c}+(k-1) T_{f}+\sum_{i=1}^{n} \text { Offset }_{i}+T_{c}} p\left(t-\left(c_{k}^{(1)}-1\right) T_{c}\right. \\
& \left.-(k-1) T_{f}-\sum_{i=1}^{n} \operatorname{Offset}_{i}\right) \times r(t) \mathrm{d} t
\end{aligned}
$$

These $L_{c}$ elementary correlations correspond to the $L_{c}$ correlations of the TPT pulses and their corresponding intervals of the IR signal. In Fig. 1, the $L_{c}$ elementary correlations are presented by the blocks indexed from 1 to $L_{c}$. $\beta$ is the input of the decision block, which in turn performs a threshold check. Hence, a match between the TPT and the IR signal is declared if the absolute value of $\beta$ exceeds certain threshold $\gamma$. Note that a (-1) output of the decision block means that a match is declared but the signal is inverted due to reflection, i.e. the corresponding $a_{l}$ is negative (see previous section).

\section{B. Example Showing the Problem with the Conventional De- tection Method}

To show the inefficiency of the conventional detection method, we present one scenario that is based on the measurement made by M. Win and R. Scholtz in [16] for an indoor 
environment. Consider a source (user 1) that is $10 \mathrm{~m}$ from the receiver. The measurement in [16] gives that the amplitude of the strongest source pulse seen by the receiver is in the order of $0.03 \mathrm{~V}$. Assume now that there is an interferer (user 2) that is $1 \mathrm{~m}$ from the receiver. The measured amplitude of the interfering pulse is of $1 \mathrm{~V}, 33$ times higher than the source pulse. Refer by $A_{r}^{(1)}\left(A_{r}^{(2)}\right.$ respectively) to the source (interferer respectively) signal amplitude at the receiver, we have $A_{r}^{(2)} \approx 33 A_{r}^{(1)}$. Let $\alpha_{0}^{(1)}\left(\alpha_{0}^{(2)}\right.$ respectively) be the output of the correlation between one source (interferer respectively) pulse and one TPT pulse when they are aligned, we can write:

$$
\alpha_{0}^{(1)}=A_{r}^{(1)} \int_{0}^{T_{c}} p^{2}(t) \mathrm{d} t=\frac{A_{r}^{(2)}}{33} \int_{0}^{T_{c}} p^{2}(t) \mathrm{d} t \approx \frac{\alpha_{0}^{(2)}}{33}
$$

$\alpha_{0}^{(2)}$ is 33 times larger than $\alpha_{0}^{(1)}$. Note that when the source signal and the TPT are perfectly aligned, neglecting the interference and noise effects, $\beta$ is equal to $L_{c} \times \alpha_{0}^{(1)}$. Consequently, $\gamma$ can not be larger than $L_{c} \times \alpha_{0}^{(1)}$, otherwise the source signal can not be detected. If $L_{c} \leq 33$, it is sufficient to have one interfering pulse aligned with one TPT pulse to get a false alarm. More explanations are given in Appendix [15].

To avoid this false alarm, but still using the conventional detection method, $L_{c}$ must be much larger than 33 , which would be an extremely unaffordable overhead in term of synchronization time, since the synchronization time is proportional to the code length $L_{c}$ [17]. Note that, when the number of concurrent transmissions increases, the situation becomes worse.

To summarize this example, the synchronization is either unfeasible or entails an extremely large overhead using the conventional detection method in none-power control IR networks when concurrent transmissions are allowed.

\section{OUR Proposal: PoWer-IndePendent Detection METHOD}

The idea behind the cross-correlation between the TPT and the IR signal is to detect a match between them. We need to find in the IR signal $L_{c}$ pulses that have the same pattern as the TPT. But the conventional detection method does not do this. It looks at the energy captured by the correlation between the TPT and the received IR signal, which is indicated by $\beta$ in Fig. 1, regardless of its distribution over the $L_{c}$ elementary correlations. So, if this energy, $\beta$, is larger than the threshold, we say that the synchronization is achieved. But what happens if all the energy comes from one elementary correlation, e.g. $\beta=\alpha_{1}$ and $\alpha_{k}=0, k=2, \ldots, L_{c}$ ? This is the challenge in the scenario shown in section III-B in the case where $L_{c} \leq 33$. Unlike the conventional detection method, the PID method solves the problem by looking at the individual energy captured by each elementary correlation separately, i.e. by looking at each $\alpha_{k}$ separately, $k=1, \ldots, L_{c}$. Fig. 2 describes the architecture of our proposal; the output of each elementary correlation $\alpha_{k}$, $k=1, \ldots, L_{c}$, passes through an elementary decision block that performs a threshold check. If the absolute value of $\alpha_{k}$ is larger than $\theta$, then a pulse is detected and the output of the elementary decision block $k$ will be 1 or -1 depending on the sign of $\alpha_{k}$ (-1 means the detected pulse has negative polarity).

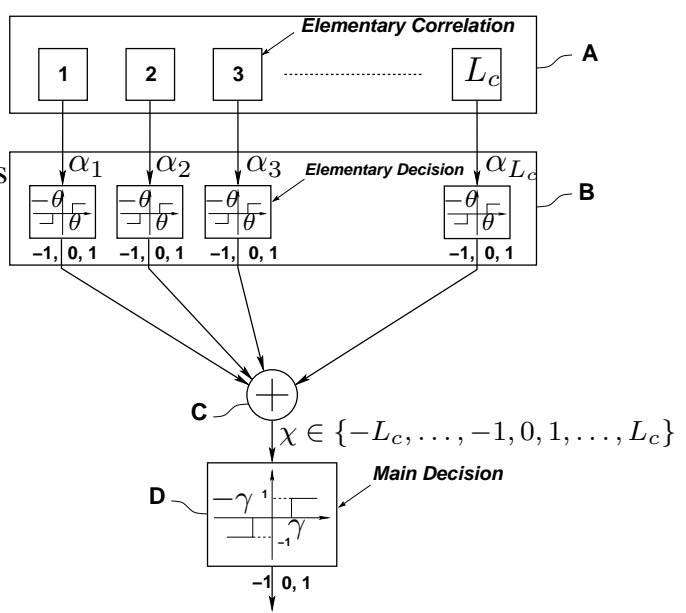

Fig. 2. PID method: each pulse is detected based on an elementary decision block. The final detection decision is based on the number of pulses detected.

Otherwise it will be 0 . Let $\chi$ be the sum of the $L_{c}$ Elementary Decision block outputs, we have:

$$
\chi=\sum_{k=1}^{L_{c}}\left(\mathbf{1}_{\left\{\alpha_{k} \geq \theta\right\}}-\mathbf{1}_{\left\{\alpha_{k} \leq-\theta\right\}}\right) \in\left\{-L_{c}, \ldots, 0, \ldots, L_{c}\right\}
$$

If the absolute value of $\chi$ is larger than the main threshold $m T h$, the output of the main decision block will be 1 or -1 (detected path is with negative polarity) and thus a match will be declared between the IR signal and the TPT. In the opposite case the output of the main decision block will be $0 . m T h$ should be a natural number less than $L_{c}$.

It is intuitively clear that this new method should solve the problem described in section III-B; it is designed for an environment without power control since it is sensitive to the existence of a pulse not to its power (assuming it has enough energy to be detected). So we call our proposal "PowerIndependent Detection".

\section{Performance Evaluation Method}

We evaluate the performance of PID and compare it to the conventional detection method.

\section{A. How to Evaluate the Performance}

For a meaningful performance evaluation of the conventional detection and the PID methods, we imbedded them in a complete synchronization method, which consists of an identification phase, followed by a verification phase. Each phase uses the two aforementioned ingredients of detection and search algorithm iteratively. For the latter, we adopted serial search. This is because we aim to evaluate the performance of the PID method independently of the impact of optimizations that use coarse synchronization.

a) The Complete Synchronization Method: When the complete synchronization method uses PID, we call it"PID synchronization method"; when it uses conventional detection, we call it "conventional synchronization method". 
Let $N$ be the number of the search bins (Appendix [15]); let "true sequence" be the sequence to be detected in the received IR signal; it has the same pattern as the TPT at the receiver.

The PID synchronization method consists of two phases (the flowchart is shown in Appendix [15]). In the first phase, the procedure in Fig. 2 without the main decision block, i.e. block $\mathrm{D}$, is repeated for the $N$ search bins according to the serial search algorithm; we start with bin 1, then bin 2 till bin $N$. The largest $\chi, \chi_{\max }$, is memorized, as well as its corresponding search bin. Then $\chi_{\max }$ is compared to a first mean threshold, mTh1. If the absolute value of $\chi_{\max }$ is strictly above $\mathrm{mTh} 1$, the bin that corresponds to $\chi_{\max }$ is considered as a signal bin, $S B$, and we move to the second phase. Otherwise, the procedure of the first phase starts anew.

In the second phase we aim to verify the detection of the first phase. It consists of $A$ iterations, in each one the procedure is the same as in the first phase but on a predefined neighborhood of $S B, V$, including $S B$, instead of the whole $N$ bins, and with a second mean threshold, mTh2, that is larger than mTh1. If at least $B$ threshold checks among $A$ succeed, the detection is confirmed, otherwise the detection of the first phase is cancelled and the procedure of the first phase starts anew.

The conventional synchronization method is similar to the PID synchronization method with the difference that it does not perform a threshold check on the elementary correlation outputs.

b) Performance Metrics: We measure the performance of each procedure by the following metrics, applied to the synchronization method: (1) the complement of the probability of Good Detection $\left(\overline{P_{G D}}=1-P_{G D}\right)$ in the presence of the true sequence in the received IR signal (2) the probability of false alarm, $P_{F A 0}$, in the absence of the true sequence in the received IR signal and (3) the total error defined as $E_{t}=\overline{P_{G D}}+P_{F A 0}$. Note that the probability of false alarm in the presence of the true sequence is included in $\overline{P_{G D}}$ and it does not give additional information about the total error, hence, for brevity, we do not consider it here as a metric.

\section{B. Computation of Metric Using Hybrid Method: Analysis + Simulation}

1) Analysis:: The goal of the analysis is to express the metrics as functions of other probabilities that we obtain by simulation. The probabilities are as follows: During the first phase we have $P_{1}$, the probability of good detection when the received IR signal contains the true sequence, and $P_{2}$, the probability of a bad detection when the received IR signal does not contain the true sequence. During the second phase we define $P_{3}$ as the probability that one threshold check succeeds, given that the first phase has resulted in a good detection in the presence of the true sequence and $P_{4}$ as the Probability that one threshold check succeeds, given that the first phase has resulted in a bad detection in the absence of the true sequence.

The Analysis presented in Appendix [15] gives:

$$
\overline{P_{G D}}=1-P_{1} \sum_{i=B}^{A}\left(\begin{array}{c}
A \\
i
\end{array}\right) P_{3}^{i}\left(1-P_{3}\right)^{(A-i)}
$$

$$
P_{F A 0}=P_{2} \sum_{i=B}^{A}\left(\begin{array}{c}
A \\
i
\end{array}\right) P_{4}^{i}\left(1-P_{4}\right)^{(A-i)}
$$

2) Simulation:: In order to compute the metrics, we ran a campaign of simulations to estimate the probabilities $P_{i}$, $i=1, \ldots, 4$. The simulations were carried out using matlab. We tried to make the simulated scenario as realistic as possible by choosing a real multipath fading channel model and by adjusting all simulation parameters, e.g. the bit energy to noise spectral density ratio $E_{0} / N_{0}$ (one bit corresponds to one pulse), the physical layer parameters, the transmission power levels, the number of users.

a) Channel Model: The simulations were carried out according to the Line of Site (LOS) indoor office channel model described in [3] for a communication range from $3 \mathrm{~m}$ to $28 \mathrm{~m}$. This model is the fruit of a campaign of industrial and academic contributions. The NLOS (Non LOS) is not considered since its parameter values are not available yet in [3].

Although the measurements made for this model were using an UWB IR signal, the model is generalized to be used by any carrier modulation system. Thus, the phase of a multipath component is considered as uniformly distributed over $[0,2 \pi]$ which is meaningless in an UWB IR baseband transmission. We solve this problem by relaxing this hypothesis and replacing it by the one adopted in [18], which is appropriate for IR baseband transmission. Then, the phase of a multipath component will be $0 / \pi$ with an equal probability for representing pulse inversion due to the reflection from different surfaces.

For simplicity, we assume that the distribution of the small scale fading is Rayleigh instead of Nakagami since the mean value in $\mathrm{dB}$ of the " $\mathrm{m}$ " parameter of the Nakagami distribution in the adopted model is very close to zero, which corresponds to the particular case of the Rayleigh distribution.

b) Simulation Parameters: We consider that all users are sending non-modulated IR signals, an assumption that does not affect our results since the interferer signals are already random with respect to the receiver and using data modulation will add one more random variable with zero mean. We have $T_{c}=$ $0.2 \mathrm{~ns}$. $N_{c}$ is set to 200 chips that corresponds to $40 \mathrm{~ns}$, which is sufficient to minimize the inter-symbol interference due the multipath delay spread in the LOS indoor office environment [19], [20], in particular we consider a guard time of 100 chips. The sampling frequency is $50 \mathrm{GHz}$, much larger than the Nyquist sampling frequency, to simulate an analog receiver since the impact of the sampling frequency is out of the scope of this study. The elementary threshold ( $\theta$ in Fig. 2 ) is set to $0.5 \times \alpha_{0}^{(1)}$ (see 8) with $A_{r}^{(1)}$ corresponds to the highest multipath components.

Each simulated scenario contains several transmitters, we refer to them as users, the one that is transmitting the true sequence is called the source and others are the interferers. The source signal power observed by the receiver is set to $-30 \mathrm{dBm}$, whereas the interferer signal powers observed by the receiver are uniformly distributed over [-30dBm, $-10 \mathrm{dBm}]$. Then the largest value of signal power that an interferer can have is $20 \mathrm{~dB}$ larger than the source signal power. Indeed, according 
to the pathloss model used in [3], this difference in power corresponds to a communication range of $17 \mathrm{~m}$ approximately (see Appendix [15]) where all users are transmitting at the same power and the source is the farthest. Such a communication range is typical for an indoor environment and the adopted channel model of [3] is still valid since it is based on measurements that cover a range from $3 \mathrm{~m}$ to $28 \mathrm{~m}$.

In all simulated scenarios, $E_{0} / N_{0}$ is computed with respect to the source signal power.

\section{Vi. Performance Evaluation Results}

\section{A. Performance Evaluation Results of the PID Synchronization Method}

The probabilities $P_{i}, i=1, \ldots, 4$, are obtained by simulation. $P_{1}$ and $P_{2}$ are computed by averaging the results of 200 independent runs for each simulated scenario. A different independent noise realization is computed per run and, within the same run, a different channel realization is computed per user.

To compute $P_{3}$ and $P_{4}$, the stationarity of the channel during the synchronization should be taken into account. Thus, the computation of $P_{3}$ and $P_{4}$ is different and more complicated. We proceed as follows: for each run of the 200 runs above, if a detection is declared, 9 other runs are done with the same channel realization for each user but with different noise realization. Then, for a given scenario, if all the 200 runs above result in a detection declaration, we will have 9 additional runs for each run and thus 1800 runs for this scenario.

We ran simulations for $E_{0} / N_{0}$ values between $0 \mathrm{~dB}$ and 20 $\mathrm{dB}, L_{c}$ values between 8 and 30 , and number of users between 5 and 20 users. In the extreme scenarios with low $E_{0} / N_{0}(<$ $10 d B)$, short $L_{c}(<16)$ and large number of users (20 users) the performance was not so good due to a huge amount of interference and noise. But, starting from $E_{0} / N_{0}=10 \mathrm{~dB}$ and $L_{c}=16$, the performance is acceptable and the results seem to be similar. For lack of space, we show only one scenario in order to explain the behavior of the PID synchronization method and to show the optimal working point.

Fig. 3 (a), (b) and (c) shows the metrics $\overline{P_{G D}}, P_{F A 0}$ and $E_{t}$ (see the legend for details). We tried to draw results for a range of $9-19$ for $\mathrm{mTh} 1$ and $\mathrm{mTh} 2$, but sometimes the values were too small (less than 10e-25) and matlab was not able to draw them. For Fig. 3 (a), the interpretation is as follows: first notice that $\overline{P_{G D}}$ is decreasing with $P_{3}$. For a given $\mathrm{mTh} 1, P_{3}$ is a decreasing function of $\mathrm{mTh} 2$, whereas $P_{1}$ is independent of mTh2. Consequently, $\overline{P_{G D}}$ is an increasing function of $\mathrm{mTh} 2$. In contrast, for a given $\mathrm{mTh} 2, P_{1}$ is a decreasing function of mTh1 but $P_{3}$ increases with mTh1 since it is a conditional probability that $\chi_{\max }$ in one iteration of the second phase is above $\mathrm{mTh} 2$ given that $\chi_{\max }$ in the first phase is above $\mathrm{mTh} 1$ and the difference between $\mathrm{mTh} 1$ and $\mathrm{mTh} 2$ decreases when mTh1 increases. Therefore, given $\mathrm{mTh} 2$, it is not evident how $\overline{P_{G D}}$ varies according to $\mathrm{mTh} 1$ since $P_{1}$ and $P_{3}$ vary in opposite directions. Moreover the values of $\mathrm{A}$ and $\mathrm{B}$ influence the impact of the variation of $P_{3}$. For $\mathrm{mTh} 2=$
$18, \overline{P_{G D}}$ increases with mTh1 when $\mathrm{mTh} 1$ goes from 12 to 17, but it decreases when mTh1 passes from 17 to 18 . Fig. 3 (b) shows the probability $P_{F A 0}$. To understand the trends of the curves, a similar interpretation can be made as above. For instance, for a given mTh1, $P_{2}$ is independent of mTh2 and $P_{4}$ is a decreasing function of mTh2. Thus, $P_{F A 0}$ decreases with $\mathrm{mTh} 2$ for a fixed mTh1. In contrast, for a fixed $\mathrm{mTh} 2, P_{2}$ is decreasing with mTh1 whereas $P_{4}$ is increasing.

Fig. 3 (c) shows $E_{t}$. The optimal working point for this scenario is for $(\mathrm{mTh} 1 ; \mathrm{mTh} 2)=(10 ; 12)$ where $E_{t}$ is minimized. On the left hand of the optimal working point, $P_{F A 0}$ is dominant and the curves imitate those of $P_{F A 0}$ in Fig. 3 (b). In contrast, $\overline{P_{G D}}$ becomes dominant on the right hand of the optimal working point and the curves at this side are similar to those of $\overline{P_{G D}}$ in Fig. 3 (a).

In conclusion, using the PID synchronization method, an optimal working point can be obtained by minimizing $E_{t}$. For this specific example, the optimal working point is $m T h 1=10$, $m T h 2=12$.

\section{B. Comparison between the PID and the Conventional Syn- chronization Methods:}

To show the performance of the conventional complete method, we computed a lower bound of its $\overline{P_{G D}}$. According to 10 , a lower bound of $\overline{P_{G D}}$ is $1-P_{1}$, which is independent of the second phase. Further, a lower bound of $1-P_{1}$ can be simply obtained by relaxing the mTh1 threshold check in the first phase (which returns as if we set mTh1 to zero). Then, a good detection is obtained if $\beta_{\max }$ ( $\chi_{\max }$ with the PID synchronization method) corresponds to a signal bin without any constraint on its value.

For the conventional complete method, the lower bound of $P_{1}$ for a given scenario is obtained by averaging the results of 30 independent runs for this scenario. Fig. 4 (a) shows two curves as functions of $E_{0} / N_{0}$ in $\mathrm{dB}$. The first curve (according to the order in the legend) corresponds to the PID synchronization method. It represents $\overline{P_{G D}}$ values at the optimal working points and that are obtained in the same way as in the section above. Corresponding values of $E_{t}$ are shown in Fig. 4 (b). The second curve gives the lower bound of $\overline{P_{G D}}$ values for the conventional complete method. The simulated scenario corresponds to 10 users, $L_{c}=20, A=10$ and $B=7$ (A and $B$ do not concern the second curve in Fig. 4 (a)). As we can notice, The PID synchronization method $\overline{P_{G D}}$ is decreasing with $E_{0} / N_{0}$ and it is very small when $E_{0} / N_{0}$ becomes larger than $10 \mathrm{~dB}$. In contrast, with the conventional complete method, $\overline{P_{G D}}$ is very high and it is of 0.97 approximately even when $E_{0} / N_{0}=20 \mathrm{~dB} . E_{t}$ in Fig. 4 (b) is a decreasing function of $E_{0} / N_{0}$, it reaches $10^{-8}$ for $E_{0} / N_{0}=15 \mathrm{~dB}$, whereas it is lower bounded by the lower bound of $\overline{P_{G D}}$, that is 0.97 , with the conventional method.

To summarize this section, we have shown that, with the PID synchronization method, the synchronization is achieved with a total error that reaches $10^{-8}$ in the shown scenarios. In contrast, with the conventional complete method, the total error is very close to 1 , which means a certain failure. The 
